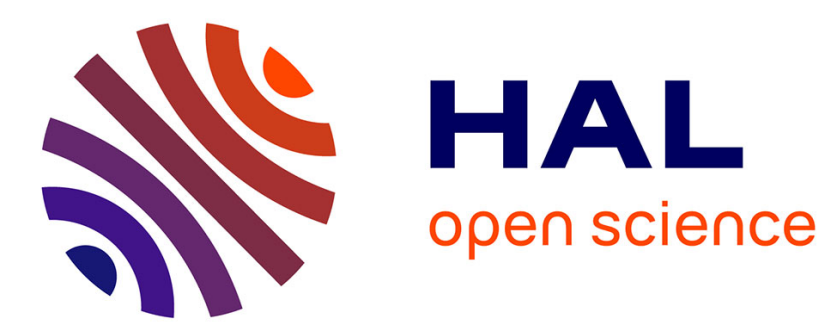

\title{
Bi-clustering continuous data with self-organizing map
}

Khalid Benabdeslem, Kais Allab

\section{To cite this version:}

Khalid Benabdeslem, Kais Allab. Bi-clustering continuous data with self-organizing map. Neural Computing and Applications, 2012, 22 (7-8), pp.1551-1562. 10.1007/s00521-012-1047-6 . hal00874676

\section{HAL Id: hal-00874676 https://hal.science/hal-00874676}

Submitted on 18 Oct 2013

HAL is a multi-disciplinary open access archive for the deposit and dissemination of scientific research documents, whether they are published or not. The documents may come from teaching and research institutions in France or abroad, or from public or private research centers.
L'archive ouverte pluridisciplinaire HAL, est destinée au dépôt et à la diffusion de documents scientifiques de niveau recherche, publiés ou non, émanant des établissements d'enseignement et de recherche français ou étrangers, des laboratoires publics ou privés. 


\title{
Bi-clustering continuous data with self-organizing map
}

\author{
Khalid Benabdeslem $\cdot$ Kais Allab
}

Received: 30 March 2011 / Accepted: 22 June 2012/Published online: 13 July 2012

(C) Springer-Verlag London Limited 2012

\begin{abstract}
In this paper, we present a new SOM-based biclustering approach for continuous data. This approach is called Bi-SOM (for Bi-clustering based on Self-Organizing Map). The main goal of bi-clustering aims to simultaneously group the rows and columns of a given data matrix. In addition, we propose in this work to deal with some issues related to this task: (1) the topological visualization of bi-clusters with respect to their neighborhood relation, (2) the optimization of these bi-clusters in macro-blocks and (3) the dimensionality reduction by eliminating noise blocks, iteratively. Finally, experiments are given over several data sets for validating our approach in comparison with other bi-clustering methods.
\end{abstract}

\section{Keywords Bi-clustering $\cdot$ SOM $\cdot$ Dimensionality} reduction

\section{Introduction}

Clustering is an important task in machine learning and data mining processes. It aims at organizing data into homogeneous groups (or clusters), such as all instances in the same group are similar to each other (cohesion property), while dissimilar from instances in other groups (separation property). This organization is only made on rows according to all columns of the data matrix. However,

K. Benabdeslem $(\bowtie)$

University of Lyon 1, LIRIS, CNRS-UMR 5205,

69622 Lyon, France

e-mail: kbenabde@univ-lyon1.fr; kbenabde@univ-lyon.fr

K. Allab

University of Lyon 1, GAMA, 69622 Lyon, France

e-mail: kais.allab@etu.univ-lyon1.fr it is clear that in the obtained partition, each class of instances is characterized according to a subset of features that participate the most on its construction. Thus, it makes good sense to simultaneously search for the specific relation that may exist between the instances and the features. This defines the block clustering (bi-clustering or crossclassification) whose purpose is to reorganize the data matrix into homogeneous blocks according to a given similarity measure.

Since the first algorithm, called Block Clustering and proposed in [17], several other bi-clustering methods have been proposed in diverse application domains, including image processing [32], text mining [10] and bioinformatics [23]. In the direct clustering approach (Block Clustering), the data matrix is divided into several sub-matrices corresponding to blocks. The division of a block depends on the variance of its values. Indeed, more the variance is low, more the block is constant. The quality of the final partition is then estimated by the sum of all the variances of these blocks [17].

In 1975, Hartigan [18] proposed two other algorithms of bi-clustering: the first one (One-Way Splitting) is mainly based on partitioning the instances with features having an intra-class variance greater than a given threshold in order to split the associated class. As any technique based on direct clustering, a minimal threshold yields a significant number of classes with low density and vice-versa. The second algorithm (Two-Way Splitting) proceeds by successive divisions of rows and columns. It calculates, at each iteration, a large number of variances. This is not adaptable to very large databases. In addition, the choice of the threshold is not obvious and requires either prior knowledge or additional tests.

In 1983, Govaert [16] proposed three algorithms of biclustering, Croeuc for continuous data, Crobin for binary 
data and Croki2 for contingency tables. The three algorithms proceed by optimizing the partitions of rows and columns by an iterative procedure based on the euclidean distance as an objective function to minimize. These algorithms can be applied to large data sets, but they require the number of both rows and columns.

The main drawback of the above algorithms is that the division (in rows and/or columns) is irreversible. In other words, these methods never reconsider the choice of a division once done. To get around this problem, Cheng and Church [7] proposed in 2000, $\delta$-clusters approach, a greedy function for creating bi-clusters by adding rows (or columns) that maximize a local gain. This approach uses the residual mean square (RMS) as similarity measure. In 2003, Yang et al. improved $\delta$-clusters by proposing another method called FLOC (Flexible Overlapped Clusters). They introduced an additional function dealing with the missing data and the overlapping [35]. In 2002, Tanay et al. [33] proposed a graph-based method called Samba, which enumerates exhaustively all the cliques modeling the possible bi-clusters in a bipartite graph from the data matrix. Other statistical models were used in an approach called Plaid Model proposed by Lazzaroni and Owen [21] and in a method of spectral bi-clustering proposed by Klugar et al. [19].

We may also cite methods of generative bi-clustering proposed by Govaert et al. [15] based on mixture models and the bi-clustering approaches proposed by Pensa et al. [27] which improve the relevance of the partitions according to background knowledge in form of constraints.

Several other methods of bi-clustering using various techniques were proposed: hierarchical bi-clustering [11], bi-clustering based on evolutionary algorithms [25], bayesian bi-clustering [24], bi-clustering using simulated annealing [5] and finally bi-clustering using random walk [1].

Furthermore, there are some methods of bi-clustering based on self-organizing maps (SOM) as DCC (Double Conjugated Clustering) [6] and KDISJ (Kohonen for Disjonctive Table) [8]. The approach DCC has problems relative to the use of two maps (one for the instances and the other for the features), which are built independently with the same dimension. KDISJ is dedicated to categorical data and will be more explained in the next section.

The aforementioned bi-clustering approaches have several shortcomings. The iterative clustering on rows and columns tries to optimize an objective function applied to one of both partitions (rows or columns) by fixing the other one. In some cases, this process of alternative optimization can be inefficient on large data sets. Indeed, the inconvenience of divisive and greedy approaches is that the decisions (division, addition/deletion of rows/columns) are taken once for all, and a bad choice could imply the loss of potential good bi-clusters [7]. Besides, the high complexity of the statistical and enumerative approaches is prohibitive. Finally, several methods require the intervention of the user to fix the values of some parameters (number of bi-clusters, thresholds, etc.).

To overcome some of these shortcomings, we propose an approach of topological bi-clustering (Bi-SOM) based on Self-Organizing Maps. SOM is a very powerful tool for analyzing and visualizing numerical data. It is used by $\mathrm{Bi}-$ SOM to cluster instances and features simultaneously in a single map and visualize the obtained blocks. In addition, Bi-SOM uses an iterative process of dimensionality reduction by eliminating blocks of irrelevant features considered as "noise".

The rest of the paper is organized as follows. The next section describes a topological approach of bi-clustering called KDISJ [8, 9]. Then, we present our proposed approach (Bi-SOM) in the third section. Finally, we provide some experimental results to validate this approach and report comparison results with other bi-clustering methods on real and synthetic data.

\section{Related work}

SOM is a very popular tool used for visualizing highdimensional data spaces. It can be considered as doing vector quantization and/or clustering while preserving the spatial ordering of the input data rejected by implementing an order of the reference vectors (also called prototype vectors, cluster centroids or reference vectors) in a one- or two-dimensional output space. The SOM consists of neurons organized on a regular low-dimensional grid, called the map.

More formally, the map is described by a graph $(\Gamma, E)$. $\Gamma$ is a set of $k$ interconnected neurons having a discreet topology defined by $E$. For each pair of neurons $(c, r)$ on the map, the distance $\delta(c, r)$ is defined as the shortest path between $c$ and $r$ on the graph. This distance imposes a neighborhood relation between neurons. Each neuron $c$ is represented by a $p$-dimensional reference vector $w^{c}=\left\{w_{1}^{c}, \ldots, w_{p}^{c}\right\}$, where $p$ is equal to the dimension of the input vectors. The number of neurons may vary from a few dozen to several thousand depending on the application.

The SOM training algorithm resembles k-means [22]. The important distinction is that in addition to the best matching reference vector, its neighbors on the map are updated. The final result is that neighboring neurons on the grid correspond to neighboring regions in the input space. The training made by SOM introduces the preservation of the topology and imposes that two nearby neurons $c, r$ by report to the discreet topology, are associated with two 


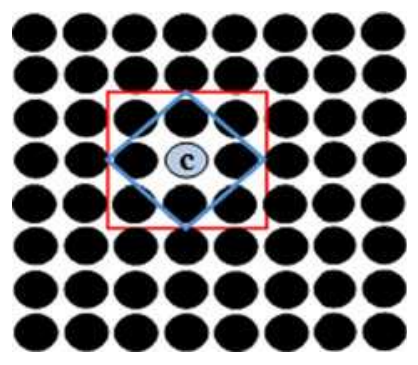

Fig. 1 Two-dimensional topological map with 1-neighborhood of a neuron $c$. Rectangular (red) with 8 neighbors and diamond (blue) with 4 neighbors (colour figure online)

nearby vectors $w^{c}, w^{r}$, by report to the distance chosen between the data (Fig. 1).

The SOM algorithm is proposed on two versions: stochastic (on-line) or batch (off-line) versions. In this paper, we choose the first version for its ability to well organize the map providing a good topology preservation from data. Theoretical comparisons between both versions can be found in [12].

The first SOM-based bi-clustering approach has been proposed by Cottrell et al. [9]. The method, called KDISJ (Kohonen for Disjonctive Table), is an extension of the algorithm KORRESP that has been introduced to analyze contingency tables [8]. The KDISJ algorithm is dedicated to categorical data and aims to simultaneously analyze the instances and the modalities of the qualitative features that describe them.

Let $A$ be a data matrix of dimension $(n \times p)$, we build the complete disjunctive table $D$ of dimension $(n \times m)$, such as $m$ is the number of the modalities of the $p$ features. $D$ is then corrected $\left(D^{c}\right)$ by the following formula:

$d_{i j}^{c}=\frac{d_{i j}}{\sqrt{d_{i . d_{. j}}}}$

where: $d_{i .}=\sum_{j=1}^{m} d_{i j} \quad$ and $\quad d_{. j}=\sum_{i=1}^{n} d_{i j}$

Note that $d_{i .}$ is equal the number of features $p$, and $d_{j}$ represents the number of instances associated with the modality $j$. After this transformation, we use the Euclidian distance on $D^{c}$ which is equivalent to use the weighted $\chi^{2}$ distance on $D$.

We then consider SOM algorithm and associate with each unit $u$ a reference vector $w^{u}$ that is comprised of $(m+n)$ components, with the first $m$ components evolving in the space of instances (represented by the rows of $D^{c}$ ) and the last $n$ components evolving in the space of modalities (represented by the columns of $D^{c}$ ). The SOM algorithm lends itself to a double learning process. At each step, we alternatively draw from $D^{c}$ a row (instance $i$ ) or a $D^{c}$ column (modality $j$ ).

When we draw an instance $i$, we associate the modality $j$ (i) defined by:

$j(i)=\arg \max _{j}\left(d_{i j}^{c}\right)=\arg \max _{j}\left(\frac{d_{i j}}{\sqrt{p d_{j}}}\right)$

that maximizes the coefficient $d_{1}^{c}$, that is the rarest modality out of all of the corresponding ones in the total population. This modality is the most characteristic for this instance. In case of ex-aequo, we do as usual in this situation, by randomly drawing a modality among the candidates. We then create an extended instance vector $X=(i, j(i))$, of dimension $(m+n)$ (Fig. 2). Subsequently, we look for the closest of all the reference vectors, in terms of the Euclidean distance restricted to the first $m$ components. Let us denote by $u^{*}$ the winning unit:

$u^{*}=\arg \min _{u}\left\|X_{(1 \ldots m)}-w_{(1 \ldots m)}^{u}\right\|^{2}$.

Next, we update the reference vector of the unit $u^{*}$ and its neighbors closer to the extended vector $X=(i, j(i))$ :

$w_{(1 \ldots m)}^{u}(t+1)=w_{(1 \ldots m)}^{u}(t)+\varepsilon \sigma\left(u, u^{*}\right)\left(X_{(1 \ldots m)}-w_{(1 \ldots m)}^{u}(t)\right)$

where $\varepsilon$ is the adaptation parameter (positive, decreasing with time) and $\sigma$ is the neighborhood function, defined by $\sigma\left(u, u^{*}\right)=1$ if $u$ and $u^{*}$ are neighbors in the Kohonen network, and $=0$ if not. The radius of the neighborhood varies in the time too.

When we draw a modality $j$ with dimension $n$ (a column of $D^{c}$ ), we do not associate an instance with it. For explaining the principle, let us denote by $Y$ (see Fig. 2) the
Fig. 2 The data matrix $D$, the corrected matrix $D^{c}$ and the vectors $X$ (line + column), $Y$ (column)

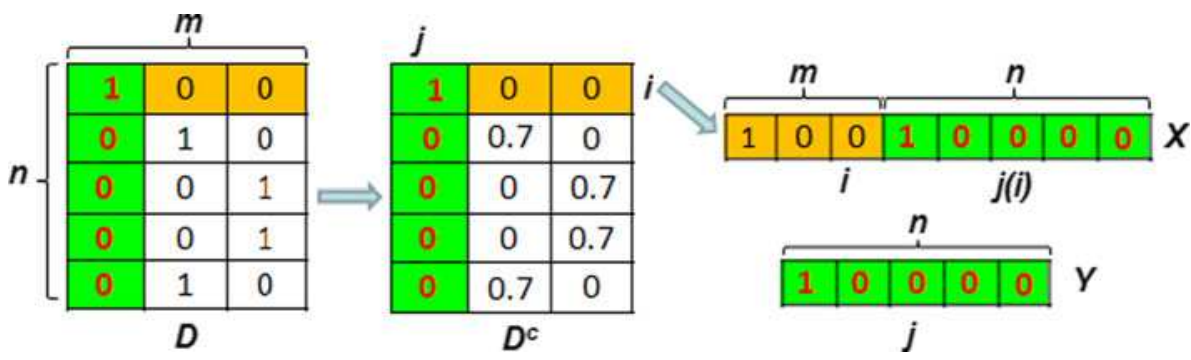


$n$-column vector corresponding to modality $j$. We then seek the closest reference vector, in terms of the Euclidean distance restricted to the last $n$ components.

Let $v^{*}$ be the winning unit:

$v^{*}=\arg \min _{u}\left\|Y_{(m+1 \ldots m+n)}-w_{(m+1 \ldots m+n)}^{u}\right\|^{2}$.

We then update the last $n$ components of the reference vector associated with $v^{*}$ and its neighbors closer to the corresponding components of the modality vector $Y$, without modifying the first $m$ components. This step can be formulated by:

$$
\begin{aligned}
w_{(m+1 \ldots m+n)}^{u}(t+1)= & w_{(m+1 \ldots m+n)}^{u}(t) \\
& +\varepsilon \sigma\left(u, v^{*}\right)\left(Y_{(m+1 \ldots m+n)}\right. \\
& \left.-w_{(m+1 \ldots m+n)}^{u}(t)\right)
\end{aligned}
$$

We so apply the SOM algorithm to the instances and modalities while maintaining them associated. Generally, $(n+m)$ iterations are enough for obtaining the convergence [9]. In the end, neighboring instances and modalities are classified in the same class or in neighboring classes. As we mentioned above, this approach handles nominal or discrete features, and therefore, continuous features need to be properly discretized. This could imply a dramatic loss of information in some cases. We suggest to solve this problem by the denition of a new link between rows and columns while respecting the nature of the continuous data.

\section{Proposed approach}

In this section, we describe our approach that we call BiSOM. The proposal carries out a bi-clustering and dimensionality reduction from a continuous data matrix. The principle of Bi-SOM, on a set of $n$ instances described by $p$ features, aims to create a partition of homogeneous data blocks; containing both instances and features that characterize them. The idea is based on this notion of characterization as the link between features and instances for clustering them simultaneously in one map.

Let $A$ be a data matrix of dimension $(n \times p)$. We consider a Kohonen network in which we associate with each unit $u$ a reference vector $w^{u}$ that consists of $(p+n)$ components. The first $p$ components represent the feature space, and the last $n$ components represent the sample space. The Kohonen algorithm lends itself to a double learning process. At each step, we alternatively draw a vector $X$ (a row or a column) from $A$.

When $X$ is a row (instance), we seek its nearest neighbor $(Y$ in $A$ ) in terms of Euclidean distance. We then create two extended vectors $V_{1}, V_{2}$ of dimension $(p+n)$ by concatenating $X$ and $Y$ with the same feature $K$ which characterizes them at best. The feature $K$ corresponds to the minimum value among the distances between the components of $X$ and those of $Y$ (Fig. 3).

$K=\arg \min _{j}\left(X_{j}-Y_{j}\right)^{2}$

Note that if there are several minimum values, we build several vectors for the same pair of instances $X$ and $Y$ with different columns for $K$.

For each vector $V_{1}$ ( $V_{2}$ respectively), we seek for the closest of all the reference vectors, in terms of the Euclidean distance using all components $(p+n)$ (contrary to KDISJ which uses the Euclidian distance restricted on the first $p$ components).

Let us denote by $u_{1}^{*}\left(u_{2}^{*}\right)$ the winning unit of $V_{1}\left(V_{2}\right)$

$u_{1}^{*}=\arg \min _{u}\left\|V_{1}-w^{u}\right\|^{2}$

Next, we update the reference vectors of the unit $u_{1}^{*}\left(u_{2}^{*}\right)$ and its neighbors in the map:

$$
\begin{aligned}
w_{(1 \ldots p+n)}^{u}(t+1)= & w_{(1 \ldots p+n)}^{u}(t)+\varepsilon \sigma\left(u, u_{1}^{*}\right)\left(V_{1(1 \ldots p+n)}\right. \\
& \left.-w_{(1 \ldots p+n)}^{u}(t)\right)
\end{aligned}
$$

Note that if $Y$ is the closest instance to $X$, and $K$ is the column which characterizes them at best, then $V_{1}=$ $\left[\begin{array}{ll}X & K\end{array}\right], V_{2}=\left[\begin{array}{ll}Y & K\end{array}\right]$ ( $\left[\begin{array}{ll}X & K\end{array}\right]$ denotes de concatenation of $X$ and $K$ ). However, $X$ may not be necessarily the closest
Fig. 3 The data matrix, the constructed vectors of dimension $(n+p): V_{1}=[X K]$ and $V_{2}=[Y K]$

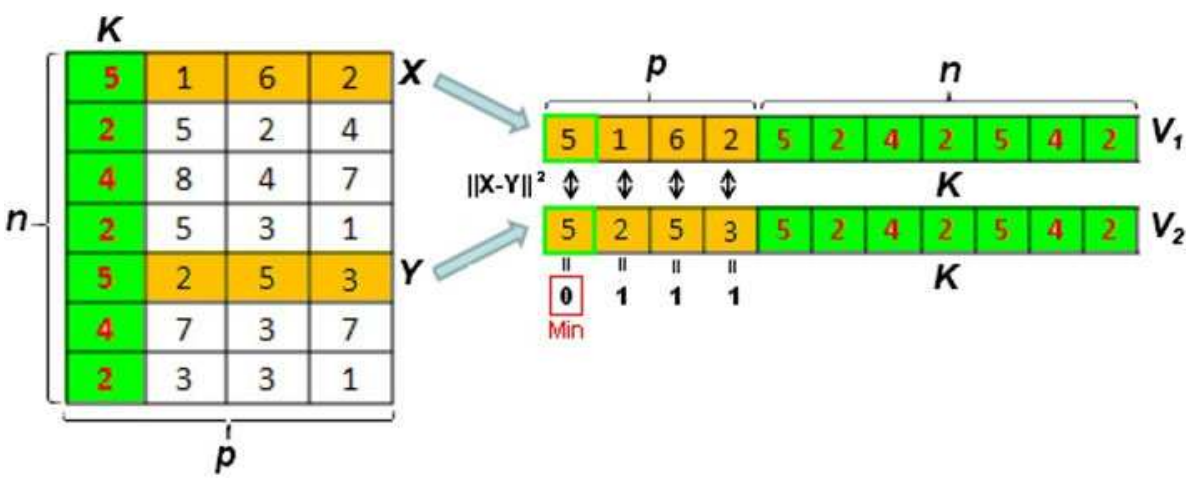


Table 1 Characteristics of used data sets

\begin{tabular}{llrrrr}
\hline Data set & Kind & $n$ & $p$ & \# class & References \\
\hline Breast & Real & 699 & 9 & 2 & {$[13]$} \\
Heart & Real & 303 & 13 & 2 & {$[13]$} \\
Ovarian & Real & 54 & 1,536 & 2 & {$[31]$} \\
Leukemia & Real & 72 & 1,762 & 2 & {$[14]$} \\
Prelic1 & Synthetic & 100 & 50 & 10 & {$[28]$} \\
Prelic2 & Synthetic & 100 & 100 & 10 & {$[28]$} \\
Prelic3 & Synthetic & 100 & 50 & 10 & {$[28]$} \\
Prelic4 & Synthetic & 100 & 100 & 10 & {$[28]$} \\
\hline
\end{tabular}

instance to $Y$. In this case, let $X^{\prime}$ be the closest instance to $Y$ and $K^{\prime}$ the feature that characterizes them at best, thus we construct $V_{3}=\left[\begin{array}{ll}Y & K^{\prime}\end{array}\right]$ and $V_{4}=\left[\begin{array}{ll}X^{\prime} & K^{\prime}\end{array}\right]$. If $V_{2}$ and $V_{3}$ are classified in different neurons, then $Y$ belongs to several classes. Consequently, this property leads the overlapping of these classes on $Y$.

When $X$ is a column (feature), with dimension $n$, we seek the reference vector (neuron) that is the closest, in terms of the Euclidean distance restricted to the last $n$ components.

Let $v^{*}$ be the winning unit of $X$ :

$v^{*}=\arg \min _{u}\left\|X_{(p+1 \ldots p+n)}-w_{(p+1 \ldots p+n)}^{u}\right\|^{2}$

We then update the last $n$ components of the winning reference vector associated with $v^{*}$ and its neighbors closer to the corresponding components of $X$, without modifying the first $p$ components:

$$
\begin{aligned}
w_{(p+1 \ldots p+n)}^{u}(t+1)= & w_{(p+1 \ldots p+n)}^{u}(t)+\varepsilon \sigma\left(u, v^{*}\right)\left(X_{(p+1 \ldots p+n)}\right. \\
& \left.-w_{(p+1 \ldots p+n)}^{u}(t)\right)
\end{aligned}
$$

In the obtained partition, a block (neuron) could be empty, containing features and instances, or containing only features. In the last case, Bi-SOM considers these features, which do not characterize no instance, as being irrelevant. Thus, they are deleted from the data matrix, and the bi-clustering is then restarted on the new data matrix. This operation is repeated until the elimination of all the blocks containing only features.

Finally, Bi-SOM is clustered by an Ascendant Hierarchical Clustering (AHC) applied on the reference vectors of the map. This Post-clustering aims at grouping together the close blocks into macro-blocks, in order to optimize their initial number.

\subsection{Algorithm}

Bi-SOM takes in input a data matrix $A$ of dimension $(n \times p)$ and returns a partition $\Gamma$ in a single topological map. The reference vectors, representing the neurons of the map, are of dimension $(p+n)$ since each vector is a result of the concatenation of a row and a column of $A$.

In the worst case, each row of $A$ will be concatenated with all the columns by building every time two vectors (the row and its nearest neighbor). Thus, the number of elements to be treated by SOM is of $2 n$ vectors (for rows) plus $p$ vectors (for columns). The complexity of SOM is $O(k n)$, where $k$ is the number of neurons in the map. Consequently, the complexity of Bi-SOM is $O(k(2 n+p))$.

Note that $B$ in the algorithm represents the "Noisy" feature set. It contains the features that do not characterize no instances in the map. In other terms, the features are considered as irrelevant if they belong to neurons having no instances. Therefore, they are deleted from the data matrix, and the bi-clustering is repeated on the new data matrix until obtaining blocks with both instances and features.

\section{Experiments}

\subsection{Data sets}

Bi-SOM is implemented in Matlab with the Toolbox available in http://www.cis.hut.fi/projects/somtoolbox/. In the first phase of experiments, we applied the approach to data describing several diseases: Breast cancer wisconsin, Heart disease, Ovarian cancer and Leukemia disease (Table 1). The Leukemia data set was used for the first time by Golub et al. [14] and by other authors afterward $[19,23]$. The data consist of 72 individuals with two types of Leukemia named ALL (Acute Lymphocytic Leukemia) and AML (Acute Myelogenous Leukemia). The data contain initially 7,129 features (genes). In [6], it was suggested to delete the "affymetrix" control-genes and the genes having a value $<20$ (biologically, the low levels of expression are difficult to interpret). Finally, 1,762 features are kept. 


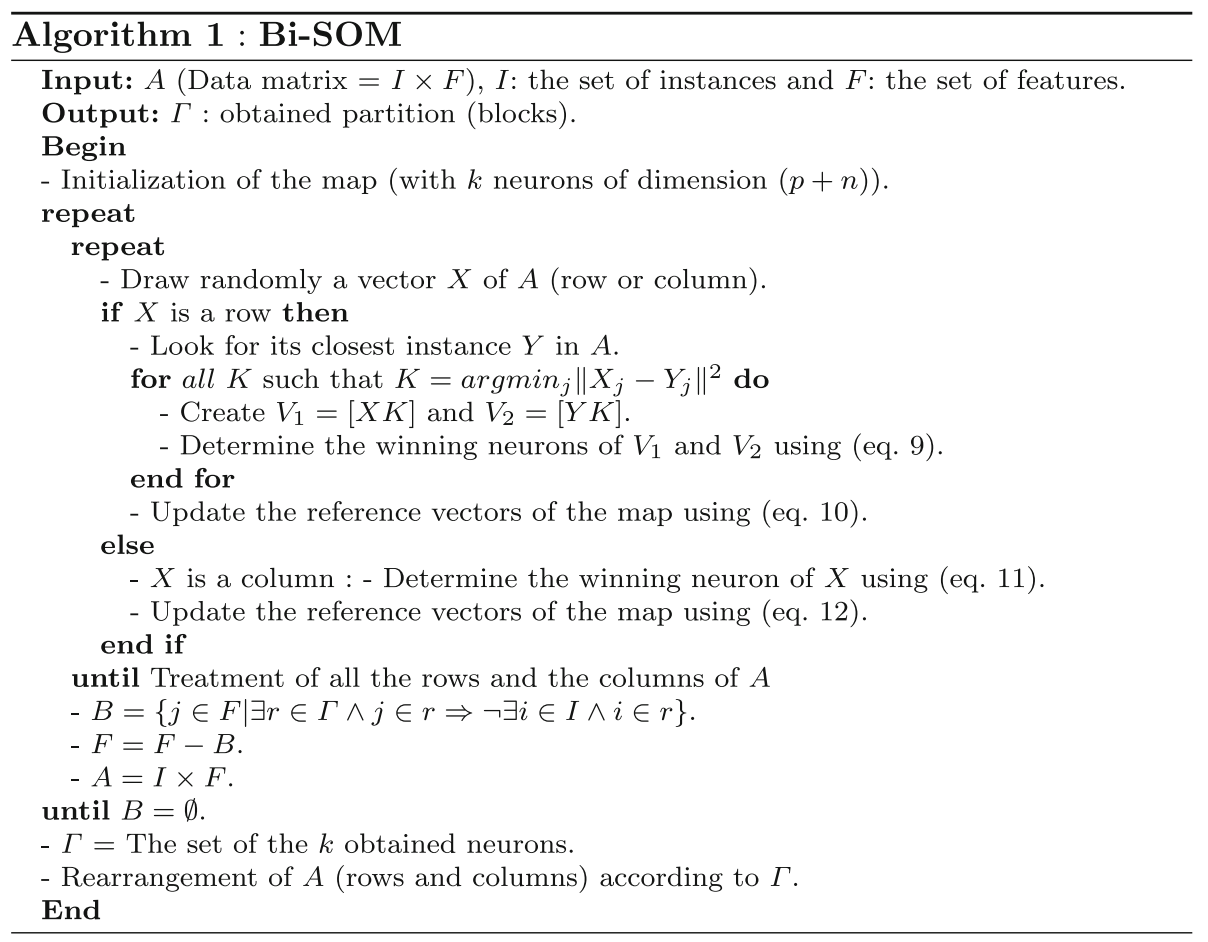

The other data sets are synthetic and provided by Prelic et al. [28] for analyzing the effects of noise and overlapping on the bi-clustering performances.

\subsection{Evaluation criteria}

It is always difficult to compare two methods of bi-clustering, because of their unsupervised nature. Nevertheless, there are some indices (internal and external) allowing to estimate the quality of the obtained partition.

\subsubsection{Internal indices}

Internal indices compare intrinsic information about data with the bi-clustering results. In this case, no a priori information further than the raw data are available [30].

An internal index is calculated from two matrices $P$ and $M$. The matrix $P$ contains the information on the proximity between elements (rows or columns) such that: $P_{i j}=$ $P_{j i}=$ distance $\left(x_{i}, x_{j}\right)$. The matrix $M$ is built as described in external indices, but inversed so higher values correspond to objects (rows or columns) not grouped together. For example $M_{i j}=1 /(1+k)$ such as $k$ is the number of times that objects $i$ and $j$ are classified together. $M_{i j}$ is included between 0 and 1 ; it is equal to 1 if $i$ and $j$ are never classified in the same class and close to 0 if they are often classified in the same class. These two matrices are compared by using the normalized Hubert index [28]:
$H(P, M)=\frac{\frac{1}{h} \sum_{i=1}^{l-1} \sum_{j=i+1}^{l}\left(P_{i j}-\mu_{P}\right)\left(M_{i j}-\mu_{M}\right)}{\sigma_{P} \sigma_{M}}$

where $l$ is the number of objects of the matrix $P$, and $h=l(l-1) / 2$. $\mu_{P}, \mu_{M}$ are the means of matrices $P$ and $M$ and $\sigma_{P}, \sigma_{M}$ their variances, respectively. We can thus calculate the index $H_{I}$ for the instances (rows) and the index $H_{F}$ for features (columns), and both indices in:

$H_{I F}=\frac{n H_{I}+p H_{F}}{n+p}$

where $n$ is the number of instances and $p$ is the number of features. The best bi-clustering is the one that minimizes the value of $H_{I F}$.

\subsubsection{External indices}

The external indices are used to compare the obtained partition with the correct partition of the data, when it is available.

Let $S$ and $R$ be two partitions, and $F$ a cross table $(S \times R) . F_{i j}$ indicates the similarity between the bi-cluster $i$ from $S$ and the bi-cluster $j$ from $R$. We use the matrix $F$ to calculate the $F$-score index [34].

- Let $g_{i}$ be the number of instances in the bi-cluster $i$ and $c_{i}$ the number of features in the bi-cluster $i\left(n_{i}=g_{i} c_{i}\right)$;

- $g_{i \cap j}$ is the number of instances belonging to both biclusters $i$ and $j$; 
- $c_{i \cap j}$ is the number of features belonging to both biclusters $i$ and $j$.

The $F$-score between $i$ and $j$ is calculated as follows:

$F$-score $(i, j)=\frac{2\left(g_{i \cap j}\right)\left(c_{i \cap j}\right)}{n_{i}+n_{j}}$

From Eq. (15), we can calculate the overall relevance between the two partitions $R$ and $S$ by:

$\operatorname{Relevance}(R, S)=\frac{1}{|R|} \sum_{i=1}^{|R|} \max _{j=1}^{|S|}(F$-score $(i, j))$

We can also use another measure to evaluate Bi-SOM by calculating the degree of similarity between the obtained partition and the correct partition (Match Score) [2]. Let $S$ and $R$ be two partitions. $G_{1}$ the set of rows in $S, G_{2}$ the set of rows in $R, C_{1}$ the set of columns in $S$ and $C_{2}$ the set of columns in $R$. The Match Score between $S$ and $R$ is:

$\operatorname{MatchScore}(S, R)=$

$$
\frac{1}{|S|} \sum_{\left(G_{1}, C_{1}\right) \in S} \max _{\left(G_{2}, C_{2}\right) \in R} \frac{\left|G_{1} \cap G_{2}\right|+\left|C_{1} \cap C_{2}\right|}{\left|G_{1} \cup G_{2}\right|+\left|C_{1} \cup C_{2}\right|}
$$

Finally, three other external indices are used: Jaccard index, Rand index (RI) [29] and Adjusted Rand index (ARI) [2]. We note by:

$a$ : the number of pairs of objects classified together in $S$ and in $R$.

$b$ : the number of pairs of objects classified together in $S$ but not in $R$.

$c$ : the number of pairs of objects classified together in $R$ but not in $S$.

$d$ : the number of pairs of objects not classified neither in $S$ nor in $R$.

$\operatorname{Jaccard}(S, R)=\frac{a}{a+b+c}$

$\mathrm{RI}(S, R)=\frac{a+d}{a+b+c+d}$

$\operatorname{ARI}(S, R)=\frac{2(a d-b c)}{(a+b)(b+d)+(a+c)(c+d)}$

\subsection{Results on synthetic data}

In the first phase of experiments, we applied Bi-SOM to synthetic data sets provided by Prelic et al. [28]. These data sets illustrate various scenarios allowing to study the effects of noise and overlapping on the proposed approach. Two types of bi-clusters are considered (constant and additive) (Table 2).
Table 2 Examples of two types of bi-clusters

\begin{tabular}{lrll}
\hline (a) Constant bi-cluster & & \\
$\mathbf{1 . 0}$ & $\mathbf{2 . 0}$ & $\mathbf{3 . 0}$ & $\mathbf{4 . 0}$ \\
1.0 & 2.0 & 3.0 & 4.0 \\
1.0 & 2.0 & 3.0 & 4.0 \\
1.0 & 2.0 & 3.0 & 4.0 \\
(b) Additive bi-cluster & & \\
$\mathbf{1 . 0}$ & $\mathbf{2 . 0}$ & $\mathbf{5 . 0}$ & $\mathbf{0 . 0}$ \\
2.0 & 3.0 & 6.0 & 1.0 \\
4.0 & 5.0 & 8.0 & 3.0 \\
5.0 & 6.0 & 9.0 & 4.0 \\
\hline
\end{tabular}

The reference rows are in bold

\subsubsection{Effect of noise}

In this phase, we applied Bi-SOM on two data sets, Prelicl (for constant bi-clusters) and Prelic2 (for additive biclusters). The aim is to analyze the sensitivity of the approach to noise, by using the Match Score as quality measure.

The first set of data Prelicl is of dimension $(100 \times 50)$. It illustrates constant bi-clusters and contains a first correct version of the data and other versions corresponding to rates of 5, 10, 15, 20 and $25 \%$ of noise. The second set of data Prelic2 is of dimension $(100 \times 100)$. It illustrates additive bi-clusters and contains a first correct version of the data, and other versions corresponding to rates of $2,4,8$ and $10 \%$ of noise.

Note that this notion of noise is different from the features's relevance study, previously described in Bi-SOM. The Noise here is imitated by adding random values drawn from a normal distribution to each cell of the original matrix.

Figure 4 shows the comparison of the results obtained by Bi-SOM with those of the methods (BiMax [28], ISA [4], Samba [33], CC [7], OPSM [3] and x-Motif [26]). In the absence of noise, Bi-SOM, ISA and Samba realize an excellent score more than $99 \%$, as well for the constant biclusters as the additive bi-clusters. BiMax realizes weak and unstable scores in the case of additive bi-clusters. The other methods have weak results for both scenarios. The most important in our case is that in the presence of the noise, Bi-SOM realizes an excellent Match Score often equal to $100 \%$ (Fig. 4), which means that the method is robust to noise, and particularly competitive to the other methods such as ISA and Samba.

Figure 5 illustrates the results obtained by Bi-SOM applied on the data Prelicl relative to the constant biclusters, with a rate of noise equal to $5 \%$. In Fig. 5 b, we show the obtained blocks (bi-clusters) after the segmentation of the map. Each block corresponds to all the neurons 

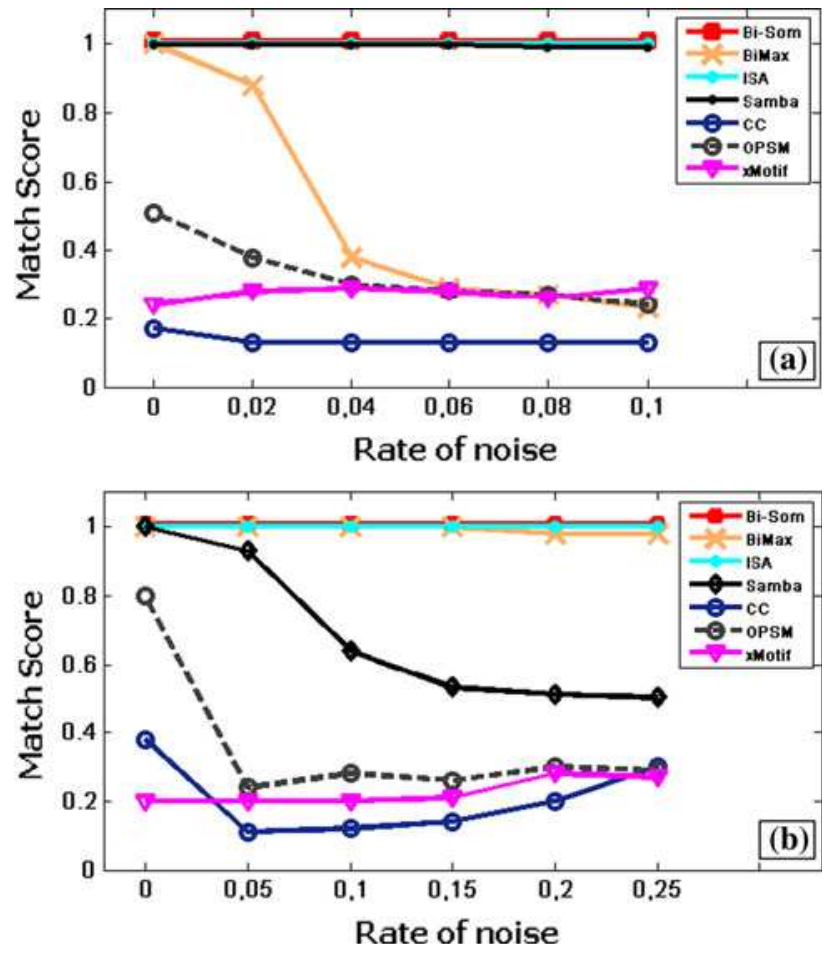

Fig. 4 Comparison between Bi-SOM and some bi-clustering methods, applied to noisy data. a Constant bi-clusters. b Additive biclusters

having the same color in Fig. 5a. We can see that Bi-SOM obtains the optimal number of ten (10) blocks, corresponding to the correct partition (10 not overlapped blocks of dimension $(10 \times 5))$. The blocks are visualized in the form of a perfect diagonal in (Fig. 5b) according to the topology illustrated in the map of Fig. 5a.

\subsubsection{Effect of overlapping}

In this phase, we applied Bi-SOM on two other data sets, Prelic3 (for constant bi-clusters) and Prelic4 (for additive bi-clusters). These data sets are not noisy but contain a first correct version of the data, and other versions containing bi-clusters overlapped in rows and columns. The rate of overlapping varies from 0 to 8 elements (rows and
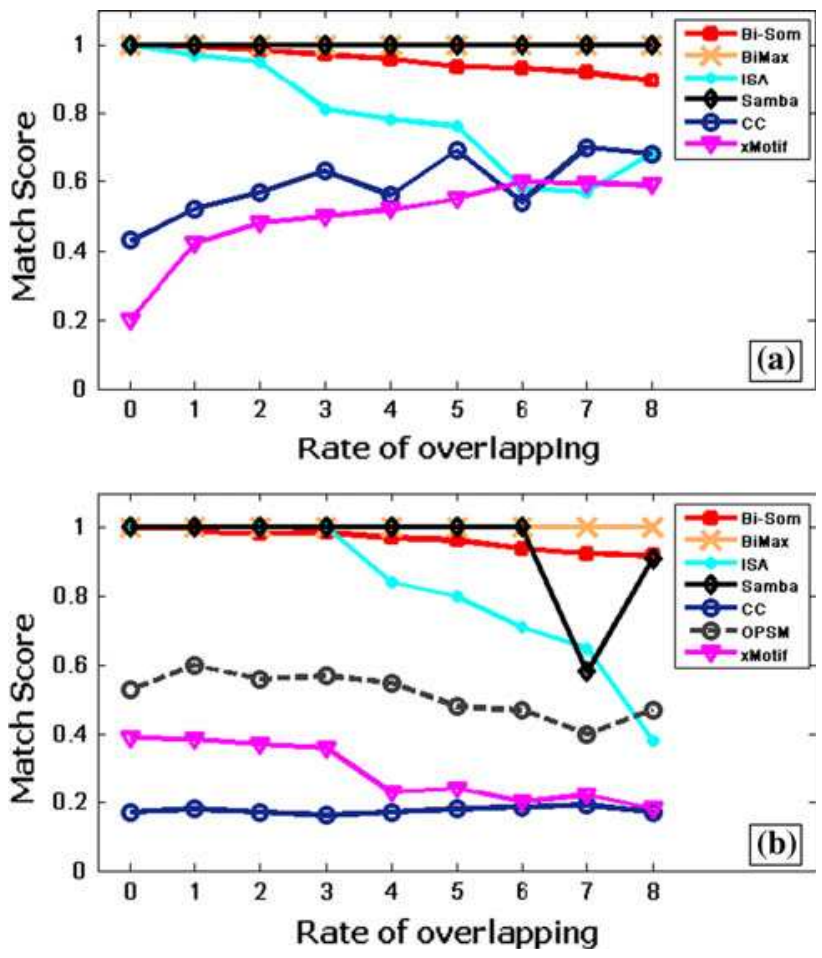

Fig. 6 Comparison between Bi-SOM and some bi-clustering methods, applied to overlapped data. a Constant bi-clusters. b Additive biclusters

columns). The goal is to analyze the sensitivity of the approach to overlapped bi-clusters.

BiMax is the only method that is stable, whatever the rate of overlapping and whatever the type of bi-clusters. Bi-SOM, Samba and ISA realize good scores, but they are sensitive to the overlapping (ISA is the most sensitive one). Although OPSM is not affected by the rate of overlapping in the case of the additive bi-clusters, it remains inefficient for the constant bi-clusters, which explains its absence in Fig. 6a.

Figure 7 shows the results obtained by Bi-SOM applied on the data Prelic4 relative to additive bi-clusters with a rate of overlapping equal to $10 \%$ (in rows and columns). Figure $7 \mathrm{~b}$ shows the obtained blocks (bi-clusters) after the segmentation of the map. Each block corresponds to all
Fig. 5 Results of Bi-SOM applied to the correct version of Prelic1 data set. a The segmented map. b The obtained blocks
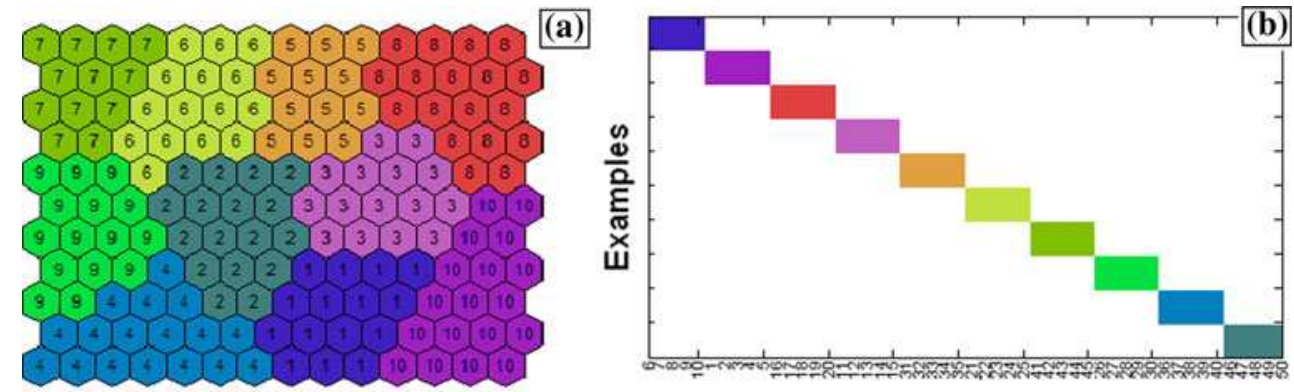

Features 
Fig. 7 Results of Bi-SOM applied to the correct version of Prelic3 data set, with a rate of overlapping equal to $10 \%$. a The segmented map. b The obtained blocks
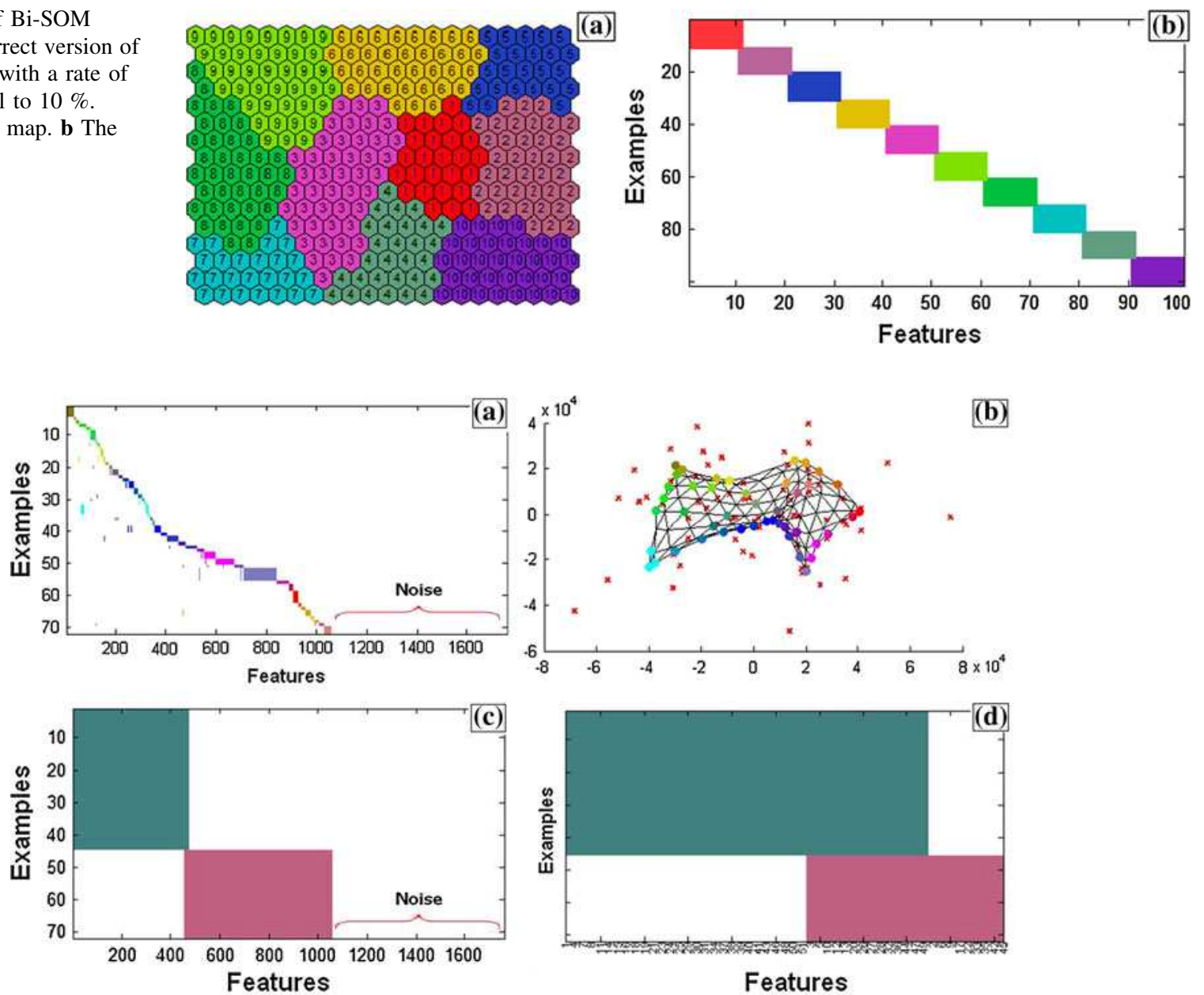

Features

$(\mathrm{e})$
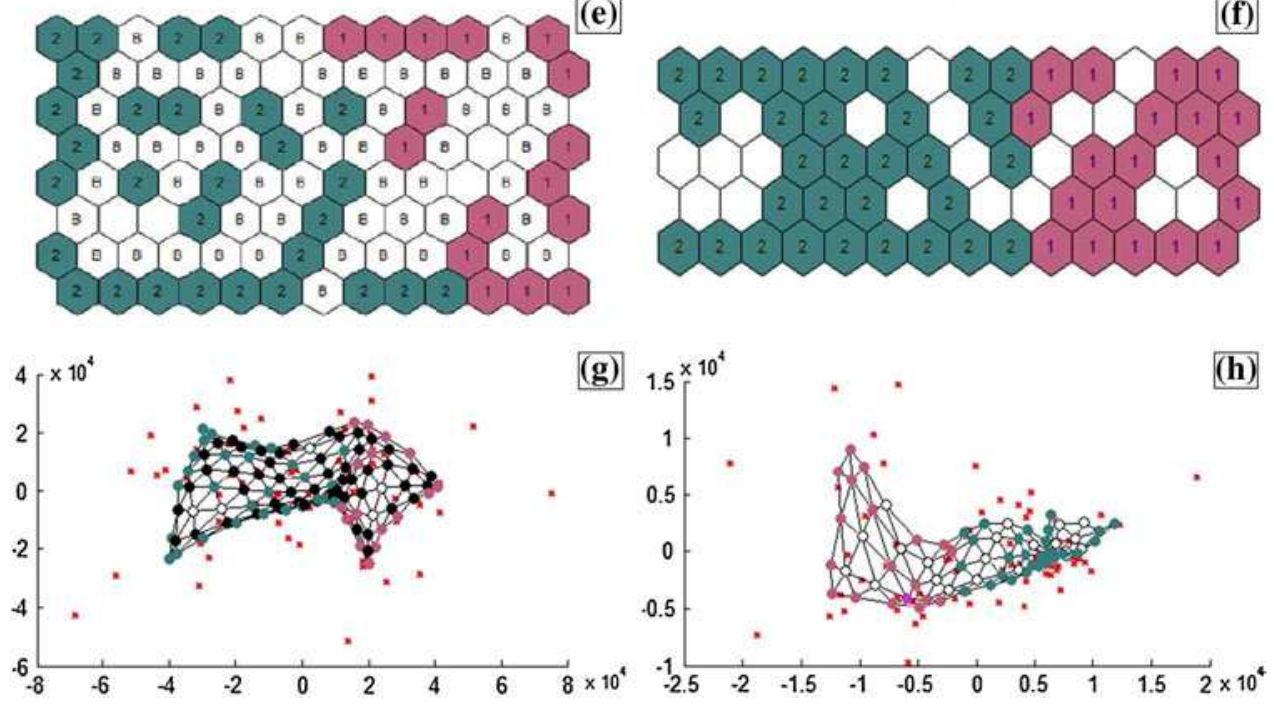

Fig. 8 Results of the bi-clustering on Leukemia data set: a the obtained blocks. b The map projected on the data space. c The obtained macro-blocks. d Macro-blocks after the reduction of

neurons having the same color in Fig. 7a. We then obtained ten (10) blocks of dimension $(10 \times 10)$, each block is overlapped by one row and one column with the block dimension. e, $\mathbf{g}$ the map optimized before the reduction of dimension. $\mathbf{f}, \mathbf{h}$ the map optimized after the reduction of dimension

which precedes it in the diagonal. That corresponds perfectly to the correct partition of this data set (Match Score $=1$ ). 


\subsection{Results on real data}

In this phase of experiments, Bi-SOM is applied on the Leukemia data set in order to visualize the results of the biclustering and the dimensionality reduction. First, we applied the Kohonen heuristic for calculating the dimensions of the map [20]. We obtained $(13 \times 8)$.

After the learning of the map, we build bi-clusters relative to neurons, and we create a binary matrix $D$, such that $D_{i j}=1$ if the row $i$ and the column $j$ are classified together, $D_{i j}=0$ otherwise. This matrix is rearranged to visualize the obtained blocks, where each block in Fig. 8a represents the neuron having the same color in Fig. 8b. After that, we apply the AHC algorithm (with "Ward" based agglomerative criterion) to group the close neurons together in macro-bloks. The Fig. 8e, g show the neurons of the map distributed in two classes (1:AML and 2:ALL) corresponding to the two macro-blocks in Fig. 8c. The Fig. 8f, $\mathrm{h}$ show the distribution of the map's neurons in two classes corresponding to the two macro-blocks in Fig. 8d after dimensionality reduction.

The neurons having the label $(B)$ represent the classes containing irrelevant features, which corresponds to the black neurons in Fig. 8g. The white neurons (without labels) in Fig. 8g, h represent the empty classes that do not contain neither instances nor features. Their existence represents the smoothing property of the SOM algorithm.

After the first learning of the map, the neurons are projected according to the distribution of the data (Fig. 8b). After the rearrangement of the binary matrix $D$, each bicluster corresponds to an active neuron which contains instances and features (Fig. 8a). We can remark that our method allows the overlapping in rows and/or columns.

The most important remark is that the white space in the right of the bi-clusters in Fig. 8a, c corresponds to the features that are not classified in classes containing instances. These features are considered as irrelevant for the bi-clustering and so eliminated from the initial data matrix. Therefore, the dimension of the map is reduced (Fig. $8 \mathrm{~g}$ before and Fig. $8 \mathrm{~h}$ after the dimensionality reduction).

For comparing our approach with the other bi-clustering approaches, we used the tool BiCAT (Bi-clustering Analysis Toolbox) available in http://www.tik.ee.ethz.ch/ sop/ bicat/. It is a tool of biological data analysis, with two known clustering methods (k-means and AHC (with "Ward" based agglomerative criterion)) and five bi-clustering approaches: (BiMax, ISA, Samba, CC, OPSM).

We applied all the methods on the real data sets (Breast, Heart, Ovarian and Leukemia). The performances of the obtained partitions are presented in Tables 3, 4, 5, 6. Note that BicAT did not detect bi-clusters by applying the method ISA on Breast data set (Table 3).
Table 3 Performances of Bi-SOM on Breast data set

\begin{tabular}{llllllll}
\hline Index & K-means & AHC & ISA & CC & OPSM & BiMax & Bi-SOM \\
\hline Jaccard & 0.242 & 0.548 & - & 0.618 & 0.511 & 0.650 & 0.884 \\
RI & 0.583 & 0.553 & - & 0.737 & 0.576 & 0.730 & 0.934 \\
ARI & 0.219 & 0.016 & - & 0.492 & 0.107 & 0.448 & 0.866 \\
Match & 0.194 & 0.137 & - & 0.173 & 0.458 & 0.049 & 0.928 \\
$\quad \begin{array}{l}\text { Score } \\
\text { Relevance }\end{array}$ & 0.105 & 0.260 & - & 0.156 & 0.136 & 0.007 & 0.963 \\
\hline
\end{tabular}

Table 4 Performances of Bi-SOM on Heart data set

\begin{tabular}{llllllll}
\hline Index & K-means & AHC & ISA & CC & OPSM & BiMax & Bi-SOM \\
\hline Jaccard & 0.118 & 0.490 & 0.500 & 0.403 & 0.502 & 0.376 & 0.547 \\
RI & 0.513 & 0.502 & 0.500 & 0.530 & 0.502 & 0.522 & 0.581 \\
ARI & 0.030 & 0.007 & 0.005 & 0.060 & 0.000 & 0.044 & 0.128 \\
Match & 0.125 & 0.114 & 0.428 & 0.114 & 0.372 & 0.227 & 0.603 \\
$\quad \begin{array}{l}\text { Score } \\
\text { Relevance }\end{array}$ & 0.061 & 0.264 & 0.267 & 0.114 & 0.124 & 0.292 & 0.683 \\
\hline
\end{tabular}

Table 5 Performances of Bi-SOM on Ovarian data set

\begin{tabular}{llllllll}
\hline Index & K-means & AHC & ISA & CC & OPSM & BiMax & Bi-SOM \\
\hline Jaccard & 0.208 & 0.453 & 0.396 & 0.497 & 0.497 & 0.585 & 0.701 \\
RI & 0.546 & 0.491 & 0.586 & 0.497 & 0.497 & 0.620 & 0.747 \\
ARI & 0.088 & 0.013 & 0.171 & 0.000 & 0.000 & 0.474 & 0.534 \\
Match & 0.150 & 0.130 & 0.394 & 0.556 & 0.324 & 0.686 & 0.898 \\
$\quad \begin{array}{l}\text { Score } \\
\text { Relevance }\end{array}$ & 0.092 & 0.259 & 0.228 & 0.133 & 0.443 & 0.814 & 0.927 \\
\hline
\end{tabular}

Table 6 Performances of Bi-SOM on Leukemia data set

\begin{tabular}{llllllll}
\hline Index & K-means & AHC & ISA & CC & OPSM & BiMax & Bi-SOM \\
\hline Jaccard & 0.204 & 0.529 & 0.515 & 0.393 & 0.520 & 0.564 & 0.540 \\
RI & 0.549 & 0.559 & 0.489 & 0.516 & 0.520 & 0.588 & 0.604 \\
ARI & 0.148 & 0.059 & 0.017 & 0.021 & 0.000 & 0.284 & 0.325 \\
Match & 0.169 & 0.162 & 0.435 & 0.099 & 0.241 & 0.662 & 0.578 \\
$\quad \begin{array}{l}\text { Score } \\
\text { Relevance }\end{array}$ & 0.118 & 0.255 & 0.163 & 0.106 & 0.145 & 0.702 & 0.681 \\
\hline
\end{tabular}

The overall results of $\mathrm{Bi}-\mathrm{SOM}$ are very encouraging on all the used indices. We can see that Bi-SOM provides partitions with a best quality in comparison with the other methods, except in some cases with the method BiMax. For example, for Leukemia data set, this method produced the best results of Jaccard index, Match score and Relevance. 
Fig. 9 Evaluation of the obtained partition of Bi-SOM for Leukemia and Ovarian data sets. a Leukemia: external indices. b Leukemia: internal index. c Ovarian: external indices. d Ovarian: internal index
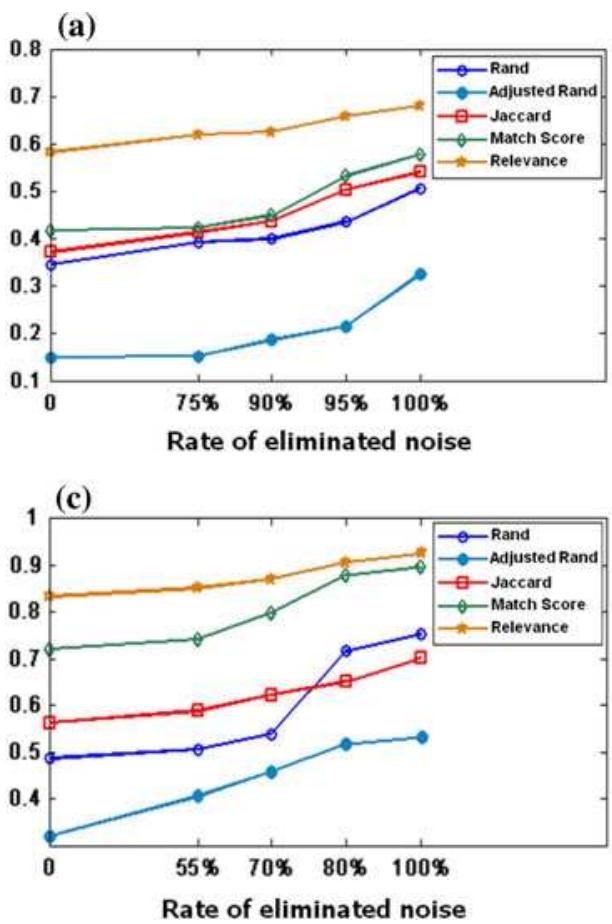

(b)

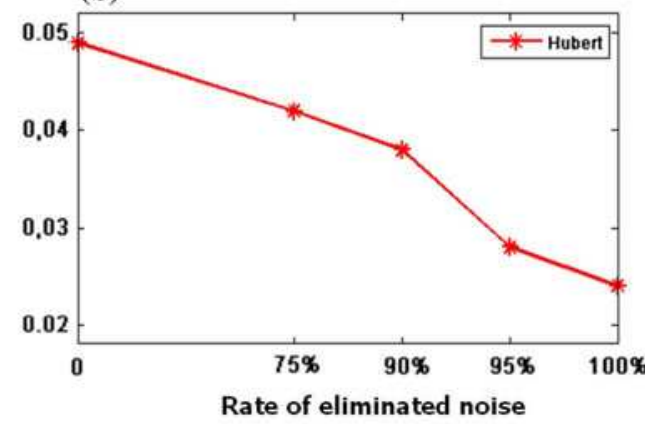

(d)

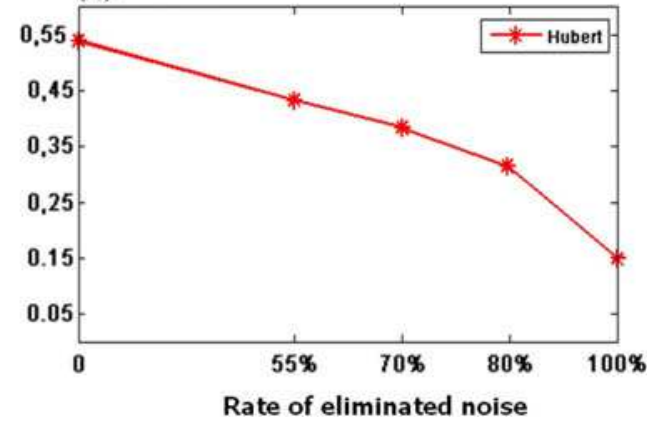

\subsection{Results of dimensionality reduction}

For Bi-SOM, a feature is irrelevant if it does not characterize no instance. In other terms, if it is classified in neurons that do not contain instances. Bi-SOM proceeds iteratively by deleting the irrelevant features and computes again the bi-clustering with the new obtained data set. This operation is repeated until deleting all irrelevant features that represent the noise. In each iteration, we calculate the performances of the obtained partition (Fig. 9).

Bi-SOM did not detect irrelevant features in both Breast and Heart data sets, which contain 9 and 13 features, respectively. However, the rate of irrelevant feature detection is of $(97 \%)$ for Leukemia and of (65\%) for Ovarian. We show in Fig. 9a, $\mathrm{c}$ that the performances of Bi-SOM are weak when it is applied to the initial data sets with irrelevant features.

The external indices (to be maximized) start with low values and increase gradually. Hence, the Hubert index (to be minimized) (Fig. 9b, d) indicates the quality of the partition obtained by Bi-SOM, by taking into account the instances and the features of bi-clusters. The obtained values are always close to 0 and show that this index is highly influenced by the deletion of the irrelevant features. Bi-SOM realizes the best performance with the totally reduced data set, (0.024) for Leukemia for (0.150) with Ovarian.

\section{Conclusion}

In this paper, we proposed a new bi-clustering approach, called Bi-SOM dedicated to continuous data. This approach is based on the definition of a new link associating with each instance, the feature that characterizes it the most. With Bi-SOM, we used the neighborhood information offered by SOM to introduce a topology between the obtained blocks. Then, we exploited this neighborhood property to optimize the obtained partition by grouping the blocks in macro-blocks.

Furthermore, Bi-SOM allowed us to reduce the dimensionality of data, by detecting and deleting the irrelevant features to improve the process of bi-clustering and consequently, the quality of the obtained partition. The comparison of the obtained results with the performances of the other biclustering methods showed that our approach is robust to noise, weakly sensitive to overlapping and remains competitive with very encouraging performance rates.

There are several avenues that could be planned for future research. First, since our proposal is sensitive to the overlapping, we could investigate in a new procedure to control this problem. Second, it would be interesting to extend the method to deal with semi-supervised problems. Finally, additional effort is needed to experiment the method on very high-dimensional data.

\section{References}

1. Angiulli F, Cesario E, Pizzuti C (2008) Random walk biclustering for microarray data. Inf Sci 178:1479-1497

2. Bandyopadhyay S, Mukhopadhyay A, Maulik U (2007) An improved algorithm for clustering gene expression data. Bioinformatics 21:2859-2865 
3. BenDor A, Chor B, Karp R, Yakhini Z (2003) Discovering local structure in gene expression data: the order preserving sub matrix problem. J Comput Biol 10(3-4):373-384

4. Bergmann S, Ihmels J, Barkai N (2004) Defining transcription modules using large-scale gene expression. Bioinformatics 20(13):1993-2003

5. Bryan K, Cunningham P, Bolshakova N (2005) Biclustering of expression data using simulated annealing. CBMS 2005:383-388

6. Busygin S, Jacobsen G, Kramer E (2002) Double conjugated clustering applied to leukemia microarray data. In: Proceedings of the 2nd SIAM international conference on data mining, workshop on clustering high dimensional data

7. Cheng Y, Church G (2000) Biclustering of expression data. In: Proceedings of the 8th international conference on intelligent systems for molecular biology (ISMB'00), vol 8, pp 93-103

8. Cottrell M, Ibbou S, Letrémy P (2004) Som-based algorithms for qualitative variables. Neural Netw 17(8-9):1149-1167

9. Cottrell M, Letrémy MP (2005) How to use the kohonen algorithm to simultaneously analyze individuals and modalities in a survey. Neurocomputing 63:193-207

10. Dhillon IS (2001) Co-clustering documents and words using bipartite spectral graph partitioning. In: Proceedings of the seventh ACM SIGKDD international conference on knowledge discovery and data mining, pp 269-274

11. Eisen M, Spellman P, Brown P, Botstein D (1998) Cluster analysis and display of genome-wide expression patterns. Proc Natl Acad Sci USA 95(25):14863-14868

12. Fort J, Cottrel M, Letrémy P (2001) Stochastic on-row algorithm versus batch algorithm for quantization and self-organizing maps. Neural networks for signal processing XI, 2001. In: Proceedings of the 2001 IEEE signal processing society workshop, pp 43-52

13. Frank A, Asuncion A (2010) UCI machine learning repository. University of California, Irvine, School of Information and Computer Sciences. http://archive.ics.uci.edu/ml

14. Golub TR, Slonim DK, Tamayo P, Huard C, Gaasenbeek M, Mesirov JP, Coller H, Loh M, Downing JR, Caligiuri MA, Bloomfield CD, Lander ES (1999) Molecular classification of cancer: class discovery and class prediction by gene expression. Science 286:531-537

15. Govaert G, Nadif M (2008) Block clustering with mixture models: comparison of different approaches. Comput Stat Data Anal 52:3233-3245

16. Govaert G (1983) Classification Croisée. Thèse d'état, Université de Paris6

17. Hartigan J (1972) Direct clustering of data matrix. J Am Stat Assoc 67(337):123-129

18. Hartigan J (1975) Direct splitting. Clustering algorithms, Chap. 14. Wiley, New York, pp 251-277
19. Klugar Y, Basri R, Chang J, Gerstein M (2003) Spectral biclustering of microarray data: coclustering genes and conditions. Genome Res 13:703-716

20. Kohonen T (2001) Self-organizing maps. Springer, Berlin

21. Lazzeroni L, Owen A (2000) Plaid models for gene expression data. Stat Sin 12:61-86

22. MacQueen J (1967) Some methods for classification and analysis of multivariate observations. Proc Fifth Berkeley Symp Math Stat Probab 1:281-297

23. Madeira S, Oliveira A (2004) Biclustering algorithms for biological data analysis: a survey. IEEE/ACM Trans Comput Biol Bioinf 1(1):24-45

24. Meeds E, Roweis S (2007) Nonparametric bayesian bi-clustering. Technical report

25. Mitra S, Banka H (2006) Multi-objective evolutionary biclustering of gene expression data. Pattern Recogn 39(12):24642477

26. Murali T, Kasif S (2003) Extracting conserved gene expression motifs from gene expression data. Pac Symp Biocomput 8:77-88

27. Pensa R, Boulicaut J-F, Cordero F, Atzori M (2010) Co-clustering numerical data under user-defined constraints. Stat Anal Data Min 3(1):38-55

28. Prelic A, Bleuler S, Zimmermann P, Wille A, Buhlmann P, Gruissem W, Hennig L, Thiele L, Zitzler E (2006) A systematic comparison and evaluation of biclustering methods for gene expression data. Bioinformatics 22(9):1122-1131

29. Rand WM (1971) Objective criteria for the evaluation of clustering methods. J Am Stat Assoc 66:846-850

30. Santamaria R, Quintales L, Theron R (2007) Methods to bicluster validation and comparison in microarray data. In: Proceedings of IDEAL 2007, LNCS4881, pp 780-789

31. Schummer M, Ng W, Bumgarner R, Nelson P, Schummer B, Bednarski D, Hassell L, Baldwin R, Karlan B, Hood L (1999) Comparative hybridization of an array of 21500 ovarian cdnas for the discovery of genes overexpressed in ovarian carcinomas. Gene 238(2):375-385

32. Shi J, Malik J (2000) Normalized cuts and image segmentation. Technical report, University of California at Berkeley, Berkeley, CA, USA

33. Tanay A, Sharan R, Shamir R (2002) Discovering statistically significant biclusters in gene expression data. Bioinformatics $18: 36-44$

34. Xiaowen L, Wang L (2007) Computing the maximum similarity bi-clusters of gene expression data. Bioinformatics 23(1):50-56

35. Yang J, Wang W, Wang H, Yu P (2003) Enhanced biclustering on expression data. BIBE '03, pp. 321-327 